\title{
Leaky barrier boosts antibiotic action
}

the

physiochemical

properties of

the $\mathrm{N}$ terminus

determine

whether a

protein can

circumvent

the macrolide

barrier.
The nascent polypeptide exit tunnel (NPET), which accommodates newly synthesized proteins as they make their way out of the bacterial ribosome, is the target of macrolide antibiotics. It was generally assumed that these drugs inhibit bacterial growth by causing a global arrest in protein synthesis; however, a new study now reveals that macrolides permit translation of a distinct subset of proteins, and that this could be even more detrimental to the cell.

Macrolides bind to a narrow region of the NPET and were previously believed to block the passage of all proteins. However, recent crystallographic studies implied that there might be enough space for a nascent peptide to squeeze through the obstructed tunnel. To investigate this further, Kannan et al. monitored ${ }^{35} \mathrm{~S}$-methionine incorporation into Escherichia coli proteins following treatment of the cells with the macrolide erythromycin or the more potent ketolide, telithromycin.

Even after exposure to 100-fold the minimum inhibitory concentration of erythromycin, protein production continued at $\sim 6 \%$ of the normal level, and strikingly, telithromycin treatment permitted protein synthesis at $\sim 25 \%$ of the normal level. Two-dimensional gel electrophoresis revealed that many of the synthesized proteins were drug specific, indicating that the chemical structure of the macrolide determined the spectrum of proteins synthesized.

So what features of the protein define its ability to by-pass the drug? Mass spectrometry of the synthesized proteins revealed that the small DNA-binding protein $\mathrm{H}-\mathrm{NS}$ was capable of by-passing all the tested macrolides. Because the amino terminus of the protein makes the initial contact with the drug during translation, this region of $\mathrm{H}-\mathrm{NS}$ was studied in more detail. When the first 18 codons of the hns gene were fused to the $5^{\prime}$ end of the osm $C$ gene (which encodes a protein that is blocked by

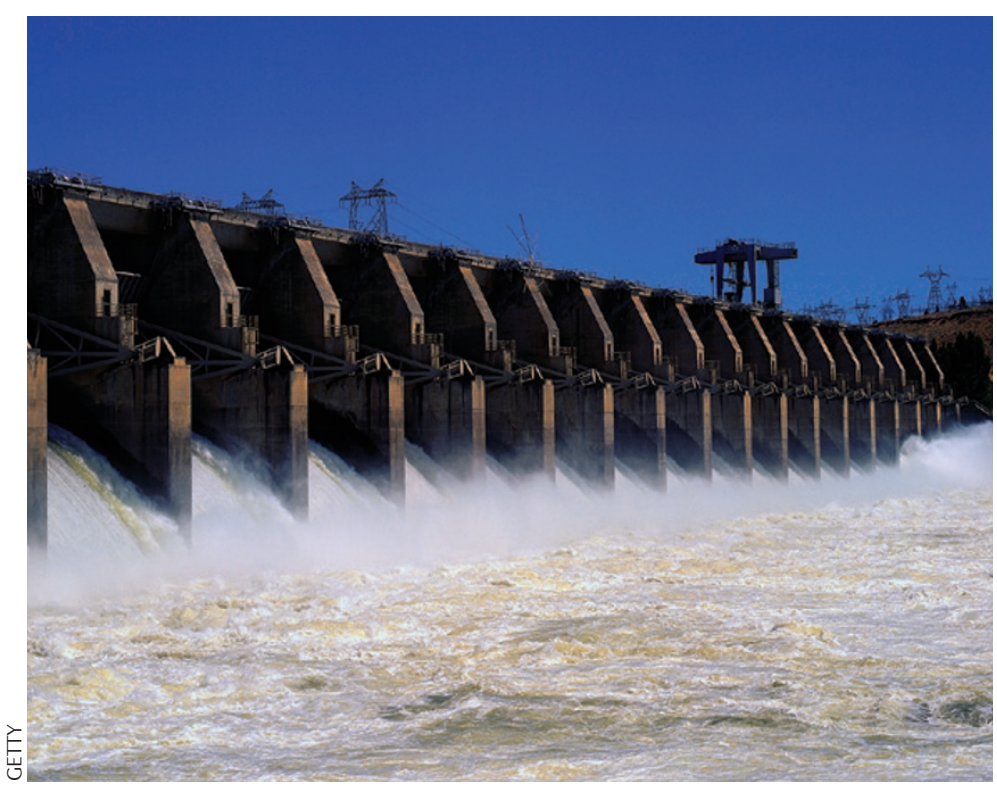

the drug), the chimeric protein bypassed erythromycin. This ability was preserved when several synonymous codon substitutions were introduced in the hns segment, showing that it is the amino acid sequence of the nascent peptide, rather than the mRNA sequence, that accounts for drug evasion. Furthermore, the protein $\mathrm{HspQ}$, which contains an $\mathrm{N}$ terminus resembling that of $\mathrm{H}-\mathrm{NS}$, was also capable of by-passing erythromycin. Together, these data suggest that the physiochemical properties of the $\mathrm{N}$ terminus determine whether a protein can circumvent the macrolide barrier.

However, not all proteins with an $\mathrm{N}$ terminus that by-passed the drug managed to escape translational arrest. In vitro translation of full-size elongation factor $\mathrm{G}$ was inhibited by telithromycin, resulting in the production of a truncated protein. Thus, the drugbound NPET retains some protein selectivity even after the $\mathrm{N}$ terminus has circumvented the drug.

These findings were not limited to $E$. coli, but were also reproduced in Staphylococcus aureus, suggesting that this is a general phenomenon. The observation that macrolides cause a selective rather than a global arrest of protein synthesis could be an important factor that influences the effectiveness of these drugs. In particular, the finding that telithromycin, which is a more potent antimicrobial than erythromycin, permits a higher level of residual protein synthesis would suggest that imbalances in the proteome are more fatal than complete shut-down of protein synthesis.

\section{Christina Tobin Kåhrström}

ORIGINAL RESEARCH PAPER Kannan, K. et al. Selective protein synthesis by ribosomes with a drug-obstructed exit tunnel. Cell 151, 508-520 (2012) 